\title{
Association of midlife value priorities with health-related quality of life, frailty and mortality among older men : a 26-year follow-up of the Helsinki Businessmen Study (HBS)
}

\section{Urtamo, Annele}

2018-05

Urtamo , A , Kautiainen , H , Pitkala , K H \& Strandberg , T E 2018 , ' Association of midlife value priorities with health-related quality of life, frailty and mortality among older men : a 26-year follow-up of the Helsinki Businessmen Study (HBS) ', Quality of Life Research, vol. 27 , no. 5 , pp. 1269-1275 . https://doi.org/10.1007/s11136-018-1805-8

http://hdl.handle.net/10138/301299

https://doi.org/10.1007/s11136-018-1805-8

unspecified

publishedVersion

Downloaded from Helda, University of Helsinki institutional repository.

This is an electronic reprint of the original article.

This reprint may differ from the original in pagination and typographic detail.

Please cite the original version. 


\title{
Association of midlife value priorities with health-related quality of life, frailty and mortality among older men: a 26-year follow-up of the Helsinki Businessmen Study (HBS)
}

\author{
Annele Urtamo ${ }^{1,2} \cdot$ Hannu Kautiainen ${ }^{1,2} \cdot$ Kaisu H. Pitkälä ${ }^{1,2} \cdot$ Timo E. Strandberg ${ }^{3,4}$
}

Accepted: 29 January 2018 / Published online: 7 February 2018

(c) Springer International Publishing AG, part of Springer Nature 2018

\begin{abstract}
Purpose Personal values influence behavior and decision making, but their long-term associations with health-related quality of life (HRQoL), frailty, and mortality are less clear. We studied these associations from midlife to old age in a 26-year follow-up of the Helsinki Businessmen Study (HBS) cohort.

Methods In 1974, 1320 clinically healthy men (born 1919-1934) reported in a 12-item questionnaire their personal values. In 2000, a mailed questionnaire, including assessment of HRQoL with RAND-36 (SF-36) instrument, was sent to survivors, and 1025 men responded. In 2000, the presence of phenotypic frailty was assessed using modified Fried criteria including indicators of shrinking, physical weakness, exhaustion, and physical inactivity. Mortality through December 31, 2000 was verified from national registries.

Results Using a factor analysis, the data of the 12-item questionnaire of personal values were loaded in 3 factors: valuing health ("Health"), enjoyable and varying life ("Enjoyment"), and comfort and work-oriented life ("Work-life-balance"). Adjusted for age, we found a significant positive association between valuing "Health" in midlife and RAND-36 domains of Physical functioning $(p=.032)$ and Vitality $(p=.005)$ in old age. "Health" also predicted less frailty $(p=.008)$, and "Enjoyment" was associated with higher mortality $(p=.017)$.

Conclusions Value priorities of men assessed in midlife had long-term associations with HRQoL and frailty in old age, and they may also predict mortality.
\end{abstract}

Keywords Personal values $\cdot$ RAND-36 $\cdot$ Functional aging $\cdot$ Prevention $\cdot$ Disability

\section{Introduction}

Personal values can be defined as the important guiding principles in life [1-3] and they drive behavior and decision making [4-6]. Schwartz's value model [2] describes human values, and the "health" value item can be located mainly in

Annele Urtamo

annele.urtamo@helsinki.fi

1 Department of General Practice and Primary Health Care, University of Helsinki, PO Box 340, 00029 Helsinki, Finland

2 Unit of Primary Health Care, Helsinki University Central Hospital, Helsinki, Finland

3 Helsinki University Hospital, University of Helsinki, Clinicum, Helsinki, Finland

4 Center for Life Course Health Research, University of Oulu, Oulu, Finland the value of security, but also in hedonism, achievement, and universalism [2, 7]. In addition, in Finnish community study [8], the Schwartz's value types of benevolence, tradition, and conformity were related positively to health, and in European study the values of benevolence and hedonism have been reported to contribute to life satisfaction [9]. Previous study has also shown that the value of security supports health behavior [10]. Furthermore, another European study indicated that openness-to-change values are associated with non-adherence to doctors' advice [11].

Consequently, health as a value is important to most individuals, and it has been recently positioned in the human values system [7]. According to this previous study, health values, in conjunction with other values, influence individual's health-related attitudes, intentions, and behaviors. Therefore, health values may associate with objective and perceived health outcomes [7]. In addition, the findings of recent cross-sectional study [12] suggested that 
giving a high value on health is related to health benefits. Furthermore, the study revealed that health as a value was associated with health-related quality of life (HRQoL), physical health, and mental health [12]. However, a longitudinal evidence on health values as determinants of health and functioning is rare.

Frailty is characterized by a decreased reserve and resistance to stressors in older people [13, 14]. A systematic review of behavioral risk factors in midlife suggested that physical activity in middle age is related to better outcomes in terms of later disability and frailty [15]. Furthermore, previous studies have shown that HRQoL, frailty $[13,14]$, and mortality in old age are adversely affected by various cardiovascular disease (CVD) risk factors [16-24], and poor self-rated health status [25] in midlife. However, the relationship between personal values and these outcomes is less clear, and deeper understanding of the longterm effects of personal values would be beneficial for health promotion and prevention of disability.

In the Helsinki Businessmen Study (HBS) cohort some personal value priorities (PVP) were reported by initially healthy participants in their midlife. In the present study, we have investigated the associations of these value priorities with HRQoL, frailty, and mortality in later life during a 26-year follow-up.

\section{Participants and methods}

\section{Study population}

Details of the HBS have been described earlier [26]. In brief, the HBS is a cohort of Finnish executives and businessmen $(N=3490)$ born between 1919 and 1934 . They had participated in volunteer health check-ups in the 1960s and early 1970s organized by Finnish Institute of Occupational Health. In 1974, the men were assessed with questionnaires, clinical, and laboratory examinations, whereupon 1815 were determined clinically healthy, and a proportion of them $(n=1222)$ participated in a primary cardiovascular prevention trial during 1974-1980 [27]. The participation in the trial did not affect the present analyses and therefore all men were included to improve statistical power.

Vital status has been monitored from national registers (Population Information System), and since 2000 mailed questionnaires have been sent regularly to survivors as described earlier [26].

The follow-up of the cohort has been approved by the ethics committee of the Department of Medicine, Helsinki University Hospital, and the study has been registered as ClinicalTrials.gov identifier: NCT02526082.

\section{Assessments in 1974}

At baseline in 1974, men were assessed by means of questionnaires and clinical and laboratory examinations. Questionnaires included items on lifestyle, such as smoking and consumption of alcohol. Weight and height were measured, and body mass index (BMI) calculated as weight (kilograms) divided by height $(\mathrm{m})$ squared. Laboratory analyses included blood pressure, serum total cholesterol, triglycerides, and glucose (fasting and post-load), and the Keys risk equation (includes age, smoking, BMI, cholesterol, and systolic blood pressure) was calculated [28].

PVP were assessed using a 12-item questionnaire, and defined as the priorities of one's subjective value system. The questionnaire was created and modified (questions of health and environment added) from Rosenberg's value assessment [29] by investigators at the Finnish Institute of Occupational Health, and a PVP scale was formed.

Personal values were assessed by asking: "How do you prioritize the following issues in your personal value system?" The items were: (1) pleasant working environment, (2) pleasant living environment, (3) job with appropriate challenges, (4) effective health services, (5) active social life, (6) exercise, (7) good possibilities for traveling, (8) variable life, (9) good health, (10) effective environmental protection, (11) long life, and (12) ability to spend a vacation at countryside. The participants were asked to evaluate all these issues using a scale as follows: $1=$ not important, $2=$ desirable, $3=$ important, $4=$ very important, $5=$ imperative. We explored various dimensions of values further by loading these items in a factor analysis. In all, PVP could be collected from 1320 out of 1815 (72.7\%) healthy participants.

\section{Assessments in $\mathbf{2 0 0 0}$}

Of the 1320 participants, 295 men died before the followup survey in 2000. Survivors were sent a mailed questionnaire which included items about demographics and lifestyle. The validated Finnish version of the RAND-36 Health Survey (similar to the Medical Outcomes Study Short-Form Survey SF-36) [30, 31], a widely used HRQoL instrument [32], was embedded in the questionnaire.

RAND-36 consists of 36 items which evaluate eight domains of HRQoL: physical functioning, role limitations caused by physical health problems, role limitations caused by emotional problems, social functioning, emotional well-being, vitality, pain, and general health perception. All scale scores range from 0 to 100, with 100 representing the most favorable state of functioning or well-being [33]. The physical functioning scale has been 
shown to be comparable to other instruments measuring disability in older adults [34], and overall RAND-36 is a reliable and valid instrument in measuring HRQoL of older people [30, 31].

The phenotypic frailty status was defined by modified Fried criteria, which have been validated in our cohort [24]. The walking speed was not measured at our study, so four following criteria were used: (1) Shrinking was based on weight loss of $\geq 5 \%$ from midlife or current BMI $<21 \mathrm{~kg} / \mathrm{m}^{2}$, (2) Physical weakness was based on self-reported difficulty carrying a grocery bag, (3) Exhaustion was based on selfreported low energy during the preceding 4 weeks (RAND36), (4) Physical inactivity was based on a question: "Do you exercise regularly weekly?" The answer "No" meant physical inactivity. The sum of fulfilled criteria classified person to be "not frail" (zero criteria), "pre-frail" (1-2 criteria), or "frail" (3-4 criteria) [24].

The analytical sample for the present study consists of men $(n=1025 ; 83.3 \%$ of survivors in 2000) who responded to questions of value priorities in 1974 and of whom HRQoL data in 2000 were available.

\section{Statistical analyses}

The data are presented as means with standard deviations (SD), as medians with interquartile range (IQR) or as counts with percentages. An exploratory factor analysis with a principal component method for factoring and oblique rotations on polychoric correlation matrix was performed to identify related items of the PVP questionnaire. The strategies used to extract the number of factors were (1) the Kaiser criteria, which determines that components with eigenvalues lower than one should be excluded, and (2) the scree test of Cattell criteria. Factor scores were computed for the rotated loading matrix using regression scoring method. Internal consistency was estimated by calculating Cronbach's alpha internal consistency with bias corrected bootstrap 95\% confidence intervals. By using adjusted (age) standardized regression coefficients, Beta $(\beta)$ linear regression analyses were performed to identify how the PVP factors scores in 1974 were associated with the HRQoL in 2000. Models included age at 2000 as covariates. $\beta$ Value is a measure of how strongly each predictor variable influences the criterion (dependent) variable, and measured in units of SD. Cohen's standard for $\beta$ values above $0.10,0.30$, and 0.50 represent small, moderate, and large relationships, respectively.

The associations between PVP factors in midlife and frailty in 2000 were analyzed using logistic regression analysis. Hazard Model was used to estimate the association of PVP with mortality (adjusted for age and Key's risk equation adjusted risk). The proportional hazards assumption was tested graphically and by use of a statistical test based on the distribution of Schoenfeld residuals. All analyses were performed using STATA 14.0 (StataCorp LP, College Station, $\mathrm{TX})$.

\section{Results}

The baseline characteristics of the cohort in 1974 are shown in Table 1. The mean age of participants in 1974 was 48 years. Of them, $28 \%$ were smokers, mean total cholesterol was $6.2 \mathrm{mmol} / \mathrm{L}$, and mean Keys' risk equation $1.99 \%$. The mean scores of personal value items are shown in Table 1 . "A good health" item got the highest score (4.4) and "an active social life" the lowest (2.1).

In the factor analysis of the 12-item value questionnaire, three factors were loaded based on the participants' scores of each question. The factors were (1) valuing health ("Health"), (2) valuing enjoyable and varying life ("Enjoyment"), and (3) valuing comfort and work-oriented life ("Work-life balance"). Of these factors, $51 \%$ were explained in this analysis. The item "an ability to spend a vacation at

Table 1 Baseline characteristics of the Helsinki Businessmen Study (HBS) sample in $1974(n=1320)$

\begin{tabular}{lc}
\hline Variable & Participants $(N=1320)$ \\
\hline Age, years, mean (SD) & $48(4)$ \\
Risk factors & \\
Current smokers, $n(\%)$ & $369(28)$ \\
Using alcohol (g/week), median (IQR) & $112(56,224)$ \\
Body mass index $\left(\mathrm{kg} / \mathrm{m}^{2}\right)$, mean (SD) & $25.8(2.8)$ \\
Blood pressure (mm/Hg), mean (SD) & \\
Systolic & $142(19)$ \\
$\quad$ Diastolic & $91(11)$ \\
Total cholesterol (mmol/L), mean (SD) & $6.2(1.1)$ \\
Triglyceride (mmol/L), mean (SD) & $1.6(0.9)$ \\
Fasting plasma glucose (mmol/L), mean & $4.7(0.7)$ \\
$\quad$ SD) & $1.99(1.81)$ \\
Keys' risk equation $(\%)$, mean (SD) & \\
Personal value priorities, mean (SD) & $4.4(0.7)$ \\
Good health & $3.9(0.8)$ \\
Job with appropriate challenges & $3.8(0.9)$ \\
Exercise & $3.8(0.8)$ \\
Pleasant living environment & $3.4(0.8)$ \\
Pleasant working environment & $3.3(1.0)$ \\
Effective health services & $3.2(1.0)$ \\
Effective environmental protection & $2.7(1.0)$ \\
Long life & $2.5(1.0)$ \\
Variable life & $2.5(1.0)$ \\
Good possibilities for traveling & $2.1(0.9)$ \\
Active social life & \\
\hline & \\
&
\end{tabular}

Keys' risk factor equation includes smoking, BMI, cholesterol, systolic blood pressure [28]

$S D$ standard deviation, IQR interquartile range 
Table 2 Explanatory factor analysis with promax-rotated factor loadings of the personal value priorities items

\begin{tabular}{|c|c|c|c|}
\hline & $\begin{array}{l}\text { Factor } 1 \\
\text { "Health" }\end{array}$ & $\begin{array}{l}\text { Factor } 2 \\
\text { "Enjoyment" }\end{array}$ & $\begin{array}{l}\text { Factor } 3 \\
\text { "Work- } \\
\text { life bal- } \\
\text { ance" }\end{array}$ \\
\hline Pleasant working environment & & & 0.81 \\
\hline Pleasant living environment & & & 0.78 \\
\hline Job with appropriate challenges & & & 0.60 \\
\hline Effective health services & 0.65 & & \\
\hline Active social life & & 0.62 & \\
\hline Exercise & 0.64 & & \\
\hline Good possibilities for traveling & & 0.79 & \\
\hline Variable life & & 0.83 & \\
\hline Good health & 0.67 & & \\
\hline $\begin{array}{l}\text { Effective environmental protec- } \\
\text { tion }\end{array}$ & 0.75 & & \\
\hline Long life & 0.43 & & \\
\hline
\end{tabular}

Coefficients with values $<0.40$ not shown. Factors explained $51 \%$ of the total variance

countryside" was left out from the analyses because it did not get enough scores to fit on the factors. The remaining factor loads are shown in Table 2. The internal consistency estimate of Cronbach's alpha was for Health 0.59 (95\% CI 0.55-0.62), for Enjoyment 0.61 (95\% CI 0.55-0.64), and for Work-life balance 0.54 (95\% CI 0.50-0.59).

In linear regression analyses, we found a significant positive association between valuing "Health" in midlife and the RAND-36 domain of Physical Functioning in old age ( $\beta 0.07,95 \%$ CI $0.01-0.14 ; p=.032$; adjusted for age) and Vitality $(\beta 0.10,95 \%$ CI $0.03-0.16 ; p=.005$; adjusted for age). There was a significant negative association between "Enjoyment" in midlife and the RAND-36 domains of Role Participation influenced by physical health problems $(\beta$ $-0.07,95 \%$ CI -0.13 to $0.00 ; p=.021$; adjusted for age) and Role Participation influenced by emotional problems ( $\beta$ $-0.06,95 \% \mathrm{CI}-0.13$ to $0.00 ; p=.032$, adjusted for age). Moreover, "Health" and RAND-36 domain of General Health tended to associate ( $\beta 0.06,95 \% \mathrm{CI}-0.00$ to 0.13 , $p=.082$ adjusted for age), and there was trend for a negative relationship between "Enjoyment" factor and RAND-36 domain of Bodily Pain $(\beta-0.05,95 \%$ CI -0.11 to 0.01 , $p=.076$ adjusted for age). Associations between factors of value priorities and HRQoL are shown in Fig. 1.

Frailty status could be assessed in 1025 men. Of those, 460 were classified as "not frail," 436 "pre-frail," and 68 "frail." We found that "Health" predicted less frailty (OR per 1-SD: $0.84,95 \%$ CI $0.75-0.95 ; p=.008$ adjusted for age), (Fig. 2a).

For mortality, there was a significant positive association between "Enjoyment" and mortality (HR per 1-SD: 1.15,

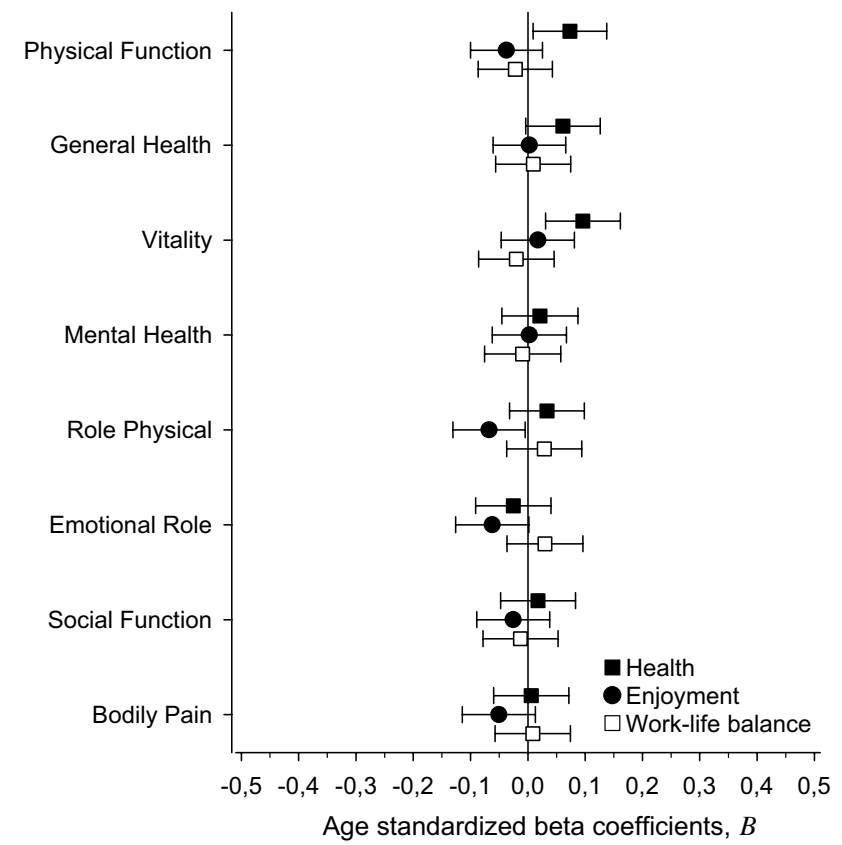

Fig. 1 Associations between factors of value priorities and HRQoL

95\% CI 1.02-1.30; $p=.017$; adjusted for age and Keys' risk equation) (Fig. 2b).

\section{Discussion}

Midlife value priorities of healthy men were associated with HRQoL and frailty in old age, and they also predicted mortality. Strikingly, valuing health in midlife ("Health" factor) was positively associated with RAND-36 domains of Physical functioning and Vitality in later life. Moreover, valuing health in midlife ("Health" factor) was associated with protection of frailty in old age. Valuing enjoyable and varying life in midlife ("Enjoyment" factor) predicted significantly lower scores in RAND-36 domains Role of Participation influenced by physical health problems and Role of Participation influenced by emotional health problems. Finally, valuing enjoyable and varying life ("Enjoyment" factor) in midlife predicted higher mortality.

The strength of our study is the very long, 26-year followup of initially healthy men. The measures used in this study are validated and widely used. However, the PVP scale was modified from its original version Rosenberg's value assessment [29] to suit better Finnish culture. One limitation is that this value assessment does not capture the full Schwartz value circle which has been used in previous studies [7-12]. However, our sample is very homogeneous representing one culture of Caucasian upper social class men. The homogeneous population is both a strength and a limitation for generalizability of the results. The businessmen had participated in 

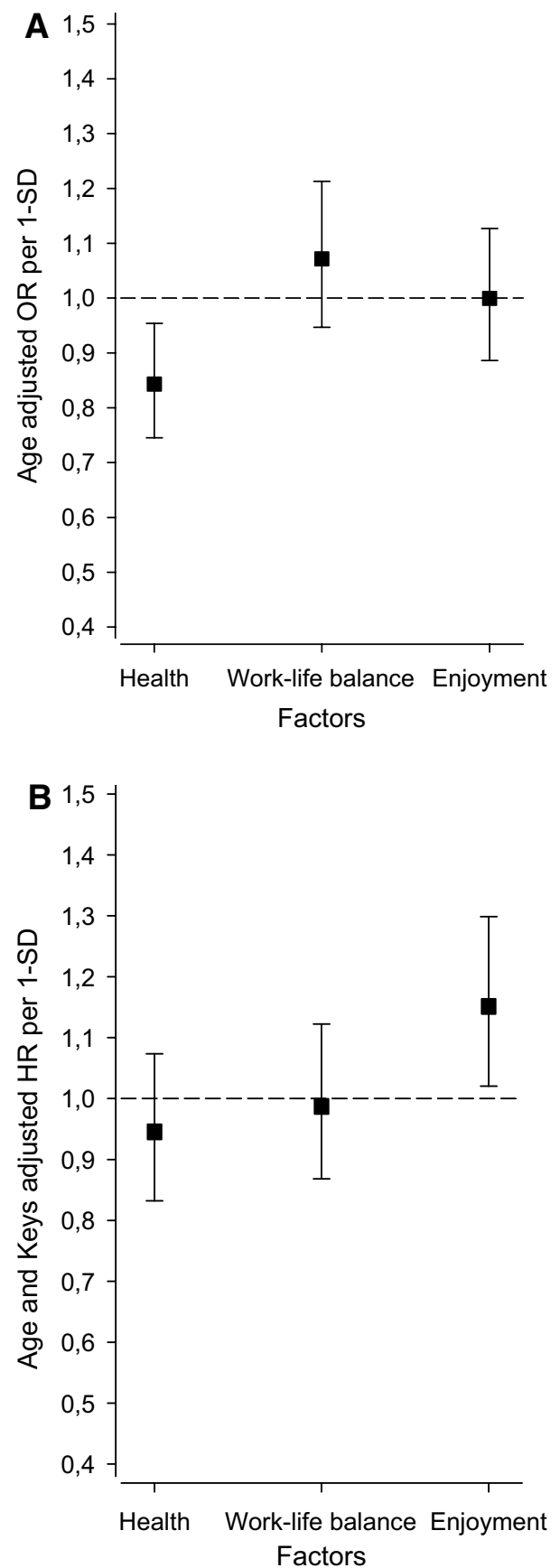

Fig. 2 a Association between factors of value priorities and frailty. b Association between factors of value priorities and mortality. Keys' risk factor equation includes smoking, BMI, cholesterol, systolic blood pressure [28]

volunteer health check-ups, so they might have been already interested in health, and that might have caused less variance compared to the general population. Another limitation was that phenotypic frailty criteria were modified from the Fried criteria. However, the present criteria have been shown to predict diseases, disability, and mortality in our cohort [24].
The assessment of PVP at baseline relied on a value assessment instrument by Rosenberg [29], which has been applied in several earlier studies of values and work [35, 36]. The questions of health and work environment were added by the original HBS investigators, and overall the instrument with its items reflects the values in the 1970s, with no questions about family values which might have been explored today. The men in our study have given values for items of each factor, and they may have given high values on several factors. Thus, the linear regression analyses shed light on how these factors ("Health," "Work-life balance," "Enjoyment") were associated with domains of HRQoL in the factor analysis. The results of our study are significant although the effects are small. The "Work-life balance" factor was less clear and meaningful than two other factors.

The associations found for factors raised a question about the differentiation between healthy and unhealthy values. Previous studies have also suggested that no particular value type is inherently healthy or unhealthy, and the values may be dependent on environment and culture [37, 38]. In our study, based on the associations of the "Enjoyment" factor, the values of enjoyable and varying life could be identified as "unhealthy" values. However, the psychological mechanism behind the association between health values and health-related quality of life, and also with other objective health outcomes, remains unclear and should be further studied. This is important because value questionnaires traditionally do not include a measure of health, which is supposed to load in the center of the Schwartz value circle and is important to most individuals [7].

To our knowledge, this is the first study to explore how personal values predict important outcomes longitudinally in old age. Although there are some studies published with much younger populations [39]. Literature is also scarce on the association between PVP and frailty. Personal values have been suggested to impact one's behavior $[4,5,7,8]$. Therefore, our results are in accordance with the systematic review [15] indicating that health behaviors in midlife are associated with successful aging and prevention of disability and frailty. Behavioral studies have proposed that subjective values influence the choices made in everyday life [6]. Previous research has also indicated that values effect behavior only if they are cognitively activated and essential to the self, which may answer the question why people do not always live up to their values [40].

One explanation for our results can be that a person valuing health attempts to make healthy choices and embraces health-related behavior. A healthy lifestyle can be determined as a comprehensive sentiment of health-related life habits and behavior [41]. Our results support the importance of promoting health at early stage, and this might be done more effectively by exploring person's values and attitudes. 
Furthermore, our results might increase understanding of long-term predictors of $\mathrm{HRQoL}$ and prevention of disability.

In conclusion, the PVP of men assessed in midlife yielded long-term associations with HRQoL and frailty in old age, and the value priorities also predicted mortality. Our findings suggest that an understanding of the long-term aspects of personal values and predictors of HRQoL can be used for health promotion and prevention of disability in old age.

Funding This study was supported by Helsinki University Hospital VTR (EVO)—funding and Academy of Finland (311492).

\section{Compliance with ethical standards}

Conflict of interest The authors have no conflict of interest to report.

Ethical approval All procedures performed in this study were in accordance with the ethical standards of the institutional research committee (Ethical approval by the Ethical committee of the Department of Medicine, Helsinki University Central Hospital) and with the 1964 Helsinki Declaration and its later amendments or comparable ethical standards.

Informed consent Informed consent was obtained from all individual participants included in the study.

\section{References}

1. Schwartz, S. H. (2012). An overview of the Schwartz theory of basic values. Online readings in Psychology and Culture. https:// doi.org/10.9707/2307-0919.1116.

2. Schwartz, S. H. (1992). Universals in the content and structure of values: Theoretical advances and empirical tests in 20 countries. In M. P. Zanna (Ed.), Advances in experimental social psychology (pp. 1-65). San Diego, CA: Academic Press.

3. Rokeach, M. (1973). The nature of human values. New York: Free Press.

4. Bardi, A., \& Schwartz, S. H. (2003). Values and behavior: Strength and structure of relations. Personality and Social Psychology Bulletin, 29(10), 1207-1220. https://doi.org/10.1177/0146167203 254602.

5. Roccas, S., Sagiv, L., Schwartz, S. H., \& Knafo, A. (2002). The big five personality factors and personal values. Personality and Social Psychology Bulletin, 28(6), 789-801. https://doi. org/10.1177/0146167202289008.

6. Feather, N. T. (1995). Values, valences, and choice: The influence of values on the perceived attractiveness and choice of alternatives. Journal of Personality and Social Psychology, 68(6), 11351151. https://doi.org/10.1037/0022-3514.68.6.1135.

7. Aavik, T., \& Dobewall, H. (2017). Where is the location of "health" in the human values system? Evidence from Estonia. Social Indicators Research, 131(3), 1145-1162. https://doi. org/10.1007/s11205-016-1287-4.

8. Sortheix, F. M., Olakivi, A., \& Helkama, K. (2013). Values, life events, and health: A study in a Finnish rural community. Journal of Community \& Applied Social Psychology, 23(4), 331-346. https://doi.org/10.1002/casp.2125.

9. Sortheix, F. M., \& Lonnqvist, J.-E. (2014). Personal value priorities and life satisfaction in Europe: The moderating role of socioeconomic development. Journal of Cross-Cultural Psychology, 45(2), 282-299. https://doi.org/10.1177/0022022113 504621.

10. Aavik, T., Aavik, A., \& Punab, M. (2014). Personal values that support and counteract utilization of a screening test for prostate cancer. Behavioral Medicine, 40(1), 22-28. https://doi. org/10.1080/08964289.2013.831805.

11. Ahola, S. (2015). Human values and non-adherence to doctors' instructions across Europe. Journal of Applied Social Psychology, 45(4), 214-225. https://doi.org/10.1111/jasp.12289.

12. Dobewall, H., Tark, R., \& Aavik, T. (2017). Health as a value and its association with health-related quality of life, mental health, physical health, and subjective well-being. Applied Research in Quality of Life, 1-14.

13. Fried, L. P., Tangen, C. M., Walston, J., Newman, A. B., Hirsch, C., Gottdiener, J., et al. (2001). Frailty in older adults: Evidence for a phenotype. The Journals of Gerontology Series A: Biological Sciences and Medical Sciences, 56(3), 146-156.

14. Rodriquez-Manas, L., Féart, C., Mann, G., Vina, J., Chatterji, S., et al. (2012). Searching for an operational definition on frailty: A Delphi method based consensus statement. The frailty operative definition - Consensus conference project. The Journals of Gerontology Series A: Biological Sciences and Medical Sciences, 68(1), 62-67. https://doi.org/10.1093/gerona/gls119.

15. Lafortune, L., Martin, S., Kelly, S., Kuhn, I., Remes, O., Cowan, A., \& Brayne, C. (2016). Behavioural risk factors in mid-life associated with successful ageing, disability, dementia and frailty in later life: A rapid systematic review. PLoS ONE, 11(2), e0144405. https://doi.org/10.1371/journal.pone.0144405.

16. Strandberg, A. Y., Strandberg, T. E., Stenholm, S., Salomaa, V. V., Pitkälä, K., \& Tilvis, R. S. (2014). Low midlife blood pressure, survival, comorbidity, and health-related quality of life in old age: The Helsinki Businessmen Study. Journal of Hypertension, 32(9), 1797-1804. https://doi.org/10.1097/HJH.0000000000000265.

17. Savela, S. L., Koistinen, P., Stenholm, S., et al. (2013). Leisuretime physical activity in midlife is related to old age frailty. The Journals of Gerontology Series A: Biological Sciences and Medical Sciences, 68(11), 1433-1438. https://doi.org/10.1093/gerona/ glt029.

18. Hyttinen, L., Strandberg, T. E., Strandberg, A. Y., et al. (2011). Effect of cholesterol on mortality and quality of life up to a 46-year follow-up. American Journal of Cardiology, 108(5), 677-681. https://doi.org/10.1016/j.amjcard.2011.04.016.

19. Strandberg, A. Y., Strandberg, T. E., Pitkälä, K., Salomaa, V. V., Tilvis, R. S., \& Miettinen, T. A. (2008). The effect of smoking in midlife on health-related quality of life in old age: A 26-year prospective study. Archives of Internal Medicine, 168(18), 19681974. https://doi.org/10.1001/archinte.168.18.1968.

20. Strandberg, T. E., Strandberg, A. Y., Salomaa, V. V., Pitkälä, K., Tilvis, R. S., \& Miettinen, T. A. (2007). The association between weight gain up to midlife, 30-year mortality, and quality of life in older men. Archives of Internal Medicine, 167(20), 2260-2261.

21. Strandberg, A. Y., Strandberg, T. E., Salomaa, V. V., Pitkälä, K., Häppölä, O., \& Miettinen, T. A. (2004). A follow-up study found that cardiovascular risk in middle age predicted mortality and quality of life in old age. Journal of Clinical Epidemiology, 57(4), 415-421. https://doi.org/10.1016/j.jclinepi.2003.09.013.

22. Strandberg, A. Y., Strandberg, T. E., Salomaa, V. V., Pitkälä, K., \& Miettinen, T. A. (2004). Alcohol consumption, 29-year total mortality, and quality of life in men in old age. The American Journal of Clinical Nutrition, 80(5), 1366-1371.

23. Strandberg, T. E., Sirola, J., Pitkälä, K. H., Tilvis, R. S., Strandberg, A. Y., \& Stenholm, S. (2012). Association of midlife obesity and cardiovascular risk with old age frailty: A 26-year follow-up of initially healthy men. International Journal of Obesity, 36(9), 1153-1157. https://doi.org/10.1038/ijo.2012.83. 
24. Sirola, J., Pitkala, K. H., Tilvis, R. S., Miettinen, T. A., \& Strandberg, T. E. (2011). Definition of frailty in older men according to questionnaire data (RAND-36/SF-36): The Helsinki Business Study. The Journal of Nutrition, Health \& Aging, 15(9), 783-787. https://doi.org/10.1007/s12603-011-0131-4.

25. Huohvanainen, E., Strandberg, A., Stenholm, S., Pitkälä, K., Tilvis, R., \& Strandberg, T. E. (2016). Association of self-rated health in midlife with mortality and old age frailty: A 26-year follow-up of initially healthy men. The Journals of Gerontology Series A: Biological Sciences and Medical Sciences, 71(7), 923-928. https://doi.org/10.1093/gerona/glv311.

26. Strandberg, T. E., Salomaa, V., Strandberg, A. Y., Vanhanen, H., Sarna, S., Pitkälä, K., et al. (2015). Cohort profile: The Helsinki Businessmen Study (HBS). International Journal of Epidemiology, 45(4), 1074-1074h. https://doi.org/10.1093/ije/dyv310.

27. Miettinen, T. A., Huttunen, J. K., Naukkarinen, V., Stranberg, T. E., Mattila, S., Kumlin, T., \& Sarna, S. (1985). Multifactorial primary prevention of cardiovascular diseases in middle-aged men: Risk factor changes, incidence, and mortality. JAMA, 254(15), 2097-2102. https://doi.org/10.1001/jama.1985.03360150073027.

28. Keys, A., Aravanis, C., Blackburn, H., et al. (1972). Probability of middle-aged men developing coronary heart disease in five years. Circulation, 45, 815-828. https://doi.org/10.1161/01. CIR.45.4.815.

29. Rosenberg, M. (1957). Occupations and values. Glecoe, IL: Free Press.

30. Haywood, K. L., Garratt, A. M., \& Fitzpatrick, R. (2005). Quality of life in older people: A structured review of generic self-assessed health instruments. Quality of Life Research, 14, 1651-1668.

31. Aalto, A.-M., Aro, A. R., \& Teperi, J. (1999). RAND-36 as a measure of health-related quality of life: Reliability, construct validity, and reference values in the Finnish general population. Helsinki: Stakes; Research Reports No.101.

32. Garratt, A., Schmidt, L., Mackintosh, A., \& Fitzpatrick, R. (2002). Quality of life measurement: Bibliographic study of patient assessed health outcome measures. BMJ, 324(7351), 1417-1422.
33. Ware, J. E., \& Kosinski, M. (2001). SF-36 physical and mental health summary scales: A manual for users of version 1 (2nd ed.). Lincoln, RI: Quality Metric Inc.

34. Dubuc, N., Haley, S. M., Ni, P., Kooyoomjian, J. T., \& Jette, A. M. (2004). Function and disability in late life: Comparison of the Late-Life Function and Disability Instrument to the ShortForm-36 and the London Handicap Scale. Disability and Rehabilitation, 26(6), 362-370.

35. Pine, G., \& Innis, G. (1987). Cultural and individual work values. The Career Development Quarterly, 35, 279-287. https://doi. org/10.1002/j.2161-0045.1987.tb00926.x.

36. Smithers, A. (1969). A structural study of the occupational value orientations of engineering students. The Vocational Aspect of Education, 21(50), 129-134. https://doi.org/10.1080/0305787698 0000211.

37. Sagiv, L., Roccas, S., \& Hazan, O. (2004). Value pathways to wellbeing: Healthy values, valued goal attainment, and environmental congruence. Positive Psychology in Practice, 68-85. https://doi. org/10.1002/9780470939338.ch5.

38. Sagiv, L., \& Schwartz, S. H. (2000). Value priorities and subjective well-being: Direct relations and congruity effects. European Journal of Social Psychology, 30(2), 177-198. https://doi. org/10.1002/(SICI)1099-0992(200003/04)30:23.0.CO;2-Z.

39. Berson, Y., \& Oreg, S. (2016). The role of school principals in shaping children's values. Psychological Science, 27(12), 1539-1549.

40. Verplanken, B., \& Holland, R. W. (2002). Motivated decision making: Effects of activation and self-centrality of values on choices and behavior. Journal of Personality and Social Psychology, 82, 434-447.

41. Abel, T. (1991). Measuring health lifestyles in a comparative analysis: Theoretical issues and empirical findings. Social Science \& Medicine, 32, 889-908. 